\title{
The transcriptional repressor Blimp-1 acts downstream of BMP signaling to generate primordial germ cells in the cricket Gryllus bimaculatus
}

\section{Citation}

Nakamura, T., and C. G. Extavour. 2016. “The Transcriptional Repressor Blimp-1 Acts Downstream of BMP Signaling to Generate Primordial Germ Cells in the Cricket Gryllus Bimaculatus." Development 143 (2) (January 15): 255-263. doi:10.1242/dev.127563.

\section{Published Version}

doi:10.1242/dev.127563

\section{Permanent link}

http://nrs.harvard.edu/urn-3:HUL.InstRepos:29386981

\section{Terms of Use}

This article was downloaded from Harvard University's DASH repository, and is made available under the terms and conditions applicable to Other Posted Material, as set forth at http:// nrs.harvard.edu/urn-3:HUL.InstRepos:dash.current.terms-of-use\#LAA

\section{Share Your Story}

The Harvard community has made this article openly available.

Please share how this access benefits you. Submit a story.

\section{Accessibility}




\title{
The transcriptional repressor Blimp-1 acts downstream of BMP signaling to generate primordial germ cells in the cricket Gryllus bimaculatus
}

\author{
Taro Nakamura ${ }^{1}$ and Cassandra G. Extavour ${ }^{1,2, *}$
}

\begin{abstract}
Segregation of the germ line from the soma is an essential event for transmission of genetic information across generations in all sexually reproducing animals. Although some well-studied systems such as Drosophila and Xenopus use maternally inherited germ determinants to specify germ cells, most animals, including mice, appear to utilize zygotic inductive cell signals to specify germ cells during later embryogenesis. Such inductive germ cell specification is thought to be an ancestral trait of Bilateria, but major questions remain as to the nature of an ancestral mechanism to induce germ cells, and how that mechanism evolved. We previously reported that BMP signalingbased germ cell induction is conserved in both the mouse Mus musculus and the cricket Gryllus bimaculatus, which is an emerging model organism for functional studies of induction-based germ cell formation. In order to gain further insight into the functional evolution of germ cell specification, here we examined the Gryllus ortholog of the transcription factor Blimp-1 (also known as Prdm1), which is a widely conserved bilaterian gene known to play a crucial role in the specification of germ cells in mice. Our functional analyses of the Gryllus Blimp-1 ortholog revealed that it is essential for Gryllus primordial germ cell development, and is regulated by upstream input from the BMP signaling pathway. This functional conservation of the epistatic relationship between BMP signaling and Blimp-1 in inductive germ cell specification between mouse and cricket supports the hypothesis that this molecular mechanism regulated primordial germ cell specification in a last common bilaterian ancestor.
\end{abstract}

KEY WORDS: Inductive signaling, Primordial germ cell, PGC, Blimp1, Prdm1, Gryllus bimaculatus, RNA interference

\section{INTRODUCTION}

The cells that make up multicellular organisms can be divided into two major categories based on the contribution of their genome to the evolutionary process: somatic cells and germ cells. Germ cells directly propagate genetic information to the next generation, and are essential for fertility as they give rise to the gametes. An important challenge for both basic biomedical and evolutionary developmental biology and for biomedical research is thus to elucidate the regulatory mechanisms regulating germ cell specification during embryogenesis. Germ cells are specified through one of two well-characterized modes, either maternally inherited germ plasm or zygotic inductive signals during

\footnotetext{
${ }^{1}$ Department of Organismic and Evolutionary Biology, Harvard University, Cambridge, MA 02138, USA. ²Department of Molecular and Cellular Biology, Harvard University, Cambridge, MA 02138, USA.

*Author for correspondence (extavour@oeb.harvard.edu)
}

Received 22 June 2015; Accepted 18 November 2015 embryogenesis (reviewed by Extavour and Akam, 2003; Extavour, 2007). In the inheritance mode, which is used by a majority of traditional laboratory model organisms including Drosophila melanogaster, Caenorhabditis elegans, Xenopus laevis and Danio rerio, maternally provided cytoplasmic determinants called germ plasm confer germ cell fate on a specific cell population in early embryos. In contrast to this inheritance mode, Mus musculus specifies germ cells through zygotic cell-cell signaling mechanisms during later embryogenesis. We previously hypothesized that, given that the inductive mode is the more widespread among metazoans, it was the ancestral mode of germ cell specification for bilaterians (Extavour and Akam, 2003). Most basally branching insects do not rely on maternally provided germ plasm to specify germ cells, in contrast to the relatively derived insect model D. melanogaster. However, experimental evidence for the inductive mode was until recently available only for two vertebrates, namely salamander and mice (reviewed by Extavour and Akam, 2003).

The two-spotted cricket Gryllus bimaculatus belongs to the basally branching group of hemimetabolous insects, and its embryonic development displays many features considered representative of ancestral arthropod embryogenesis (Donoughe and Extavour, 2015). G. bimaculatus is also an emerging model organism for functional genetic comparative developmental studies (Mito and Noji, 2009), including analysis of germ line development (Ewen-Campen et al., 2013; Donoughe et al., 2014). We recently reported that $G$. bimaculatus primordial germ cell (PGC) formation requires bone morphogenetic protein (BMP) signaling, thus demonstrating conservation of this mechanism between mice and cricket (Donoughe et al., 2014). However, how BMP signals activate downstream genes to specify germ cells in G. bimaculatus is still unknown. Furthermore, if the BMP-based germ cell specification mechanisms in mice and crickets were homologous, then we would expect conservation of the molecular mechanisms operating downstream of BMP activation in both systems. Obtaining experimental evidence addressing this hypothesis is important to address the question of whether a single, ancestral PGC specification mechanism is likely to have operated in a last common bilaterian ancestor.

In mice, two key transcriptional repressors have been identified as key factors that operate downstream of BMP signaling to specify germ cell fate (Ohinata et al., 2005; Yamaji et al., 2008): Prdm1 (also commonly referred to as Blimp1) and Prdm14. Both of these genes encode proteins that contain a positive regulatory domain [the PR domain, related to the Suppressor of variegation 3-9, Enhancer of zeste, and Trithorax (SET) methyltransferase domain] and five Krüppel-type $\mathrm{C} 2 \mathrm{H} 2$ zinc-finger ( $\mathrm{ZF}$ ) domains (Seetharam and Stuart, 2013). Blimp-1 was first identified as a repressor of interferon- $\beta$ gene expression (Turner et al., 1994). In mouse 
embryos, Blimp-1 and Prdm 14 are initially expressed in a few cells of the proximal posterior epiblast, where PGCs arise at E6.25 to E6.5 in response to Bmp4 signals emanating from the extraembryonic ectoderm (Lawson et al., 1999). The Blimp-1/Prdm14expressing cells become PGCs (Ohinata et al., 2005, 2009; Yamaji et al., 2008); this fate acquisition includes repression of somatic differentiation programs (Saitou et al., 2002) and epigenetic reprogramming of the genome (Seki et al., 2007). Blimp-1- or Prdm14-deficient embryos display impaired PGC specification (Ohinata et al., 2005; Yamaji et al., 2008). Thus, Blimp-1 and Prdm14 are key BMP-dependent regulators for generating PGCs in mice.

The Prdm gene family first emerged in metazoans (Seetharam and Stuart, 2013). Blimp-1 orthologs have been identified in many bilaterian animals, including D. melanogaster ( $\mathrm{Ng}$ et al., 2006; Agawa et al., 2007; Akagi and Ueda, 2011), rabbit (Hopf et al., 2011), D. rerio (Baxendale et al., 2004; Wilm and Solnica-Krezel, 2005), X. laevis (de Souza et al., 1999), salamander (Chatfield et al., 2014), Gallus gallus (Wan et al., 2014), lamprey (Nikitina et al., 2011), sea star (Hinman and Davidson, 2003; Fresques et al., 2014) and sea urchin (Wang et al., 1996; Livi and Davidson, 2006; Fresques et al., 2014). Consistent with evidence from mice, Blimp-1 is widely expressed in a variety of endodermal and/or mesodermal tissues in these animals. However, in axolotl, an animal known to utilize the inductive mode for specifying PGCs (Nieuwkoop, 1951), Blimp-1 transcripts are not expressed in the lateral mesoderm where PGCs arise, and expression of Bmp4 does not induce Blimp-1 expression in animal cap assays (Chatfield et al., 2014). This suggests that, although a role for BMP signaling in PGC specification is conserved across a number of animals, the molecular mechanisms downstream of BMP signaling in this process may have diverged, at least within vertebrates. Functional experiments examining the roles of Blimp-1 in embryogenesis and organogenesis have been reported in other species (see Discussion), but a role in PGC specification has not been reported in animals other than mice. The extent to which Blimp-1mediated PGC specification is a conserved feature of BMP-based PGC specification in animals thus remains an open question.

In this study, we examined the expression patterns and functions of the Blimp-1 ortholog Gb-Blimp-1 in the cricket G. bimaculatus. We show that $G b$-Blimp-1 transcripts are expressed in mesodermal tissues in the abdominal segments where PGCs first arise. Our functional analyses revealed that $G b$-Blimp- 1 transcript levels are determined by BMP signaling input, and that Gb-Blimp-1 is essential for $G$. bimaculatus PGC development. We therefore provide the first evidence for functional conservation of the role of BMP-dependent Blimp-1 expression in germ cell specification across protostomes and deuterostomes, suggesting that this function might have been present in the last common ancestor of Bilateria.

\section{RESULTS}

\section{Cloning of the cricket G. bimaculatus homolog of Blimp-1}

In order to investigate whether Blimp-1-mediated germ cell induction operates in G. bimaculatus, we first searched for Prdm family genes using $G$. bimaculatus embryonic transcriptome data (Zeng et al., 2013; T. Bando, personal communication). We found a single near full-length sequence with both of the highly conserved domains that are characteristic of Blimp-1 genes, namely a PR domain and five ZF domains (Fig. 1A). We assessed the identity of the gene using phylogenetic analysis (Fig. S1A), and identified conserved residues between this $G$. bimaculatus sequence and Blimp-1 orthologs from other animals (Fig. S1B). Based on these data, we concluded that Gb-Blimp-1 is the G. bimaculatus homolog

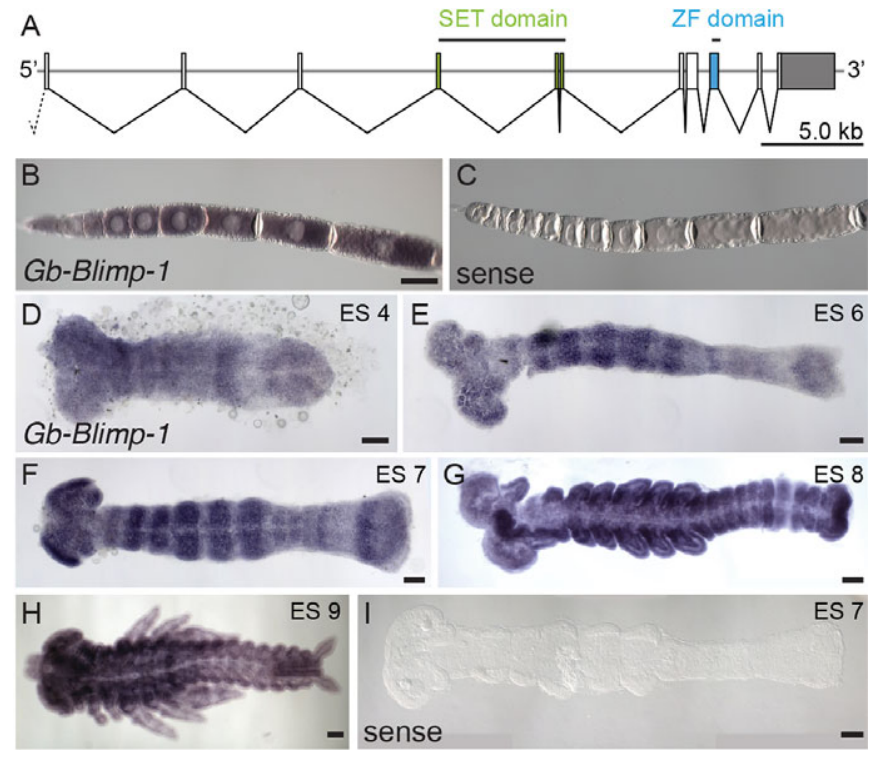

Fig. 1. Gene structure and expression patterns of the Gryllus homolog of Blimp-1 during oogenesis and embryogenesis. (A) Schematic representation of gene and protein structures of the Gryllus Blimp-1 ortholog Gb-Blimp-1. Gb-Blimp-1 contains the conserved SET domain (green) and a zinc-finger (ZF, blue) domain. The $3^{\prime}$-UTR is in gray. Regions used to synthesize dsRNA and RNA probe are indicated by black lines above the SET and ZF domains, respectively. (B,C) Expression of Gb-Blimp-1 during oogenesis. (C) Sense probe for oogenesis in situ hybridization. Gb-Blimp-1 is expressed throughout the ooplasm at all stages of embryogenesis but not in the follicle cells or terminal filaments of the adult ovariole. (D-H) Expression of Gb-Blimp-1 during embryogenesis at the indicated embryonic stages (ES). Gb-Blimp-1 is expressed in spatially restricted domains in abdominal segments during PGC specification stages (ES6-6.5). (I) Sense probe for embryogenesis in situ hybridization. Scale bars: $100 \mu \mathrm{m}$ (B also applies to $\mathrm{C}$ ).

of mammalian Blimp-1 (Prdm1). We also identified short fragments covering the $\mathrm{C} 2 \mathrm{H} 2$ domains of putative $G$. bimaculatus orthologs of the D. melanogaster Prdm genes Prdm3 and Prdm16 (hamlet). However, neither the transcriptome nor the draft $G$. bimaculatus genome (T. Mito, personal communication) contained a clear G. bimaculatus ortholog of the vertebrate Prdm14 gene. As no ortholog of Prdm14 has been identified in any arthropod, it is possible that $\operatorname{Prdm} 14$ is the result of a deuterostome-specific duplication.

\section{Gb-Blimp-1 is expressed dynamically during germ band elongation}

In situ hybridization indicated that Gb-Blimp-1 is expressed dynamically in a variety of tissues and organs during oogenesis and embryogenesis. In the ovaries of sexually mature $G$. bimaculatus adults, we detected Gb-Blimp-1 transcripts in oocytes of all stages of oogenesis, with no asymmetrical localization apparent at any stage (Fig. 1B,C). We did not detect expression in terminal filament cells, follicle cells or the oocyte nucleus (Fig. 1B,C).

During embryogenesis, abdominal segments are specified sequentially from anterior to posterior, and appear to arise from a posteriorly located growth zone (Mito et al., 2010). We previously showed that Gb-piwi transcripts, a conserved molecular marker for PGCs, are first observed at high levels in small subsets of cells in the lateral mesoderm of abdominal segments A2-A4 during the posterior elongation process at embryonic stages (ES) 6-7 (EwenCampen et al., 2013; stages here and throughout as per Donoughe and Extavour, 2015). We therefore asked whether Gb-Blimp-1 was 
expressed at the time and place of PGC specification. In contrast to mouse Blimp- 1 expression, which is observed in only a few cells of the proximal posterior epiblast where PGCs arise (Ohinata et al., 2005), we found that Gb-Blimp-1 transcripts are present ubiquitously during embryogenesis, and that short-lived enriched expression appears as broad stripes in the mesodermal tissues of some segments at the germ band stage (Fig. 1D-G, Fig. S2). At ES4 [42 $\mathrm{h}$ after egg laying (AEL)], prior to the onset of PGC specification, we detected Gb-Blimp-1 transcripts throughout the entire embryo, but with enriched expression in broad stripes in the gnathal and thoracic segments (Fig. 1D). By ES6 (60 h AEL), at the beginning of PGC specification, Gb-Blimp-1 expression levels appear to increase in segmental stripes in the mesodermal tissues of the gnathal, thoracic and first abdominal segments (Fig. 1E, Fig. S2A-B"). In addition to the enriched expression domains in these anterior abdominal segments, we detected newly arising expression domains in the ectoderm and mesoderm adjacent to the anteriormost part of the posterior unsegmented region (the so-called 'growth zone') (Fig. 1E, Fig. S2C-D"). At ES7, the segmental expression domains in the gnathal and thoracic mesoderm became further refined into more clearly defined striped patterns (Fig. 1F,I, Fig. S2E,F). In the abdomen, the region of strongest mesodermal expression at this stage is no longer $\mathrm{A} 1$, but rather A2-A3, the segments where the largest number of PGCs arise (Fig. 1F,I, Fig. 2A-A", Fig. S2E-F"). Consistent with the Gb-Blimp-1 transcript expression pattern, we detected Gb-Blimp-1 protein at ES7 in the mesoderm throughout the thorax and abdomen (Fig. S3A-A"), including the mesodermal tissue of segments A2-A3 (Fig. S3C-C"). The specificity of the cross-reactive anti-Blimp-1 antibody used in the latter experiments was confirmed with RNA interference (RNAi) against Gb-Blimp-1 (Fig. S3F-G').

$\mathrm{By}$ the end of posterior segment elongation and PGC cluster formation (ES8-8.5, 72-96 h AEL), Gb-Blimp-1 transcripts in the abdomen were no longer detected in a striped pattern, but instead appeared ubiquitous and uniform. At this stage, we detected enriched expression of Gb-Blimp-1 transcripts and protein in the mesodermal tissues of thoracic appendage primordia, in the labrum and in the hindgut primordium (Fig. 1G, Fig. 2B-B", Fig. S3B-B",E-E"). Interestingly, unlike at earlier stages, at ES8 the expression patterns of Gb-Blimp-1 transcript and protein no longer exactly corresponded: we detected both in the mesodermal cells of thoracic leg primordia (Fig. 1G, Fig. 2B-B", Fig. S3B-B",E-E"), but did not detect Gb-Blimp-1 protein in the mesodermal cells of the thoracic segments (Fig. S3E-E"). Furthermore, co-staining for nuclei revealed that Gb-Blimp-1 expression was enriched in the abdominal coelomic pouches [structures of mesodermal origin in all segments posterior to the head, which lie laterally and dorsally to PGCs clusters (Ewen-Campen et al., 2013)], but appeared absent from the abdominal ectoderm (Fig. 2B-B"). At ES9 (4.5 days AEL), when appendage formation is almost completed, we detected continued Gb-Blimp-1 expression in the mesodermal tissues of all appendage primordia and in the coelomic pouches of each abdominal segment (Fig. 1H, Fig. S3F,F'). Taken together, these expression data suggested that $G b$-Blimp- 1 could play a role in the formation of germ cells.

We aimed to verify that the Gb-Blimp-1 expression in the abdominal mesodermal cells at ES6-7 was indeed present in the developing germ cells. Since PGCs are specified by BMP signals (Donoughe et al., 2014), we first performed co-staining of the BMP signal effector phosphorylated Mad (pMad) and Gb-Blimp-1 transcripts. In contrast to nuclear pMad, which is detected at highest levels dorsally and at lower levels ventrally (Donoughe
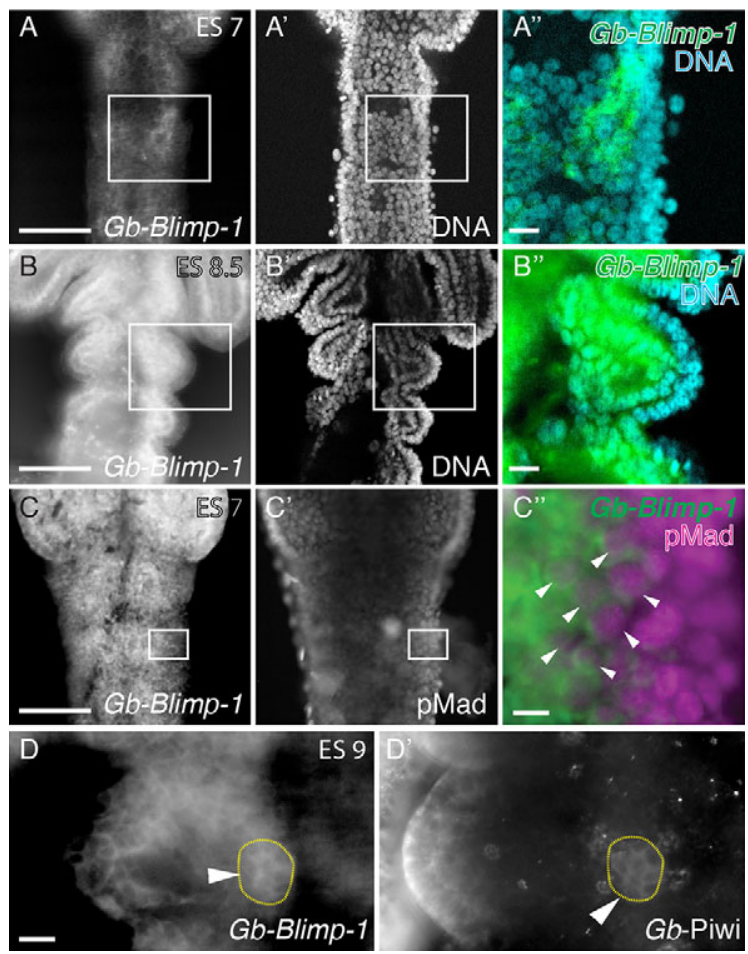

Fig. 2. Expression of Gb-Blimp-1 transcripts during Gryllus PGC formation. (A-B") In situ hybridization for Gb-Blimp-1 and nuclear staining at ES7 and ES8.5 (early PGC specification stages) show that Gb-Blimp-1 is expressed in the mesoderm but excluded from the ectoderm. (A-A") At ES7, Gb-Blimp-1 expression is highest in A2 segmental mesoderm but also present at lower levels throughout the mesoderm in the rest of the embryo. (B-B") At ES8.5, the highest expression levels are detected in abdominal coelomic pouches, which are mesodermal structures. (C-C") Co-staining of Gb-Blimp-1 transcripts and pMad protein reveals that BMP signals are transduced by $\mathrm{Gb}$ Blimp-1-expressing mesodermal cells in PGC origin regions. Arrowheads $\left(\mathrm{C}^{\prime \prime}\right)$ indicate examples of cells containing both nuclear pMad and Gb-Blimp-1 transcripts. Gb-Blimp-1 expression is highest in medial (ventral) mesodermal cells and is absent from lateral (dorsal) mesodermal and ectodermal cells. As previously reported (Donoughe et al., 2014), nuclear pMad signals are highest in the dorsal mesoderm and ectoderm. (D, $\left.\mathrm{D}^{\prime}\right)$ Comparison of Gb-Blimp-1 transcripts (D) and Gb-Piwi protein ( $\mathrm{D}^{\prime}$ ) expression domains reveals Gb-Blimp1 signals in PGCs at ES9. Arrowheads and yellow circles indicate a cluster of PGCs in A3. DNA is labeled with Hoescht 33342. Anterior abdominal segments (A1-A3) are shown in A-C'. Boxed regions are magnified in $A^{\prime \prime}-C^{\prime \prime}$. Scale bars: $100 \mu \mathrm{m}$, except $10 \mu \mathrm{m}$ in $\mathrm{A}^{\prime \prime}-\mathrm{C}^{\prime \prime}$.

et al., 2014), Gb-Blimp-1 transcripts were detectable at highest levels ventrally and appeared to be absent in the ectoderm and mesoderm in the dorsalmost regions (Fig. 2C). However, we detected co-expression of Gb-Blimp-1 transcripts and nuclear localized pMad signals in a subset of mid-dorsally located mesodermal cells (Fig. 2C"), suggesting that there is active BMP signaling in some Gb-Blimp-1-expressing cells that are located in the same position as nascent PGCs. At subsequent stages (ES9), when PGCs form clusters adjacent to the coelomic pouches, $G b$ Blimp-1 transcripts were detected co-expressed with Gb-Piwi protein in PGC clusters (Fig. 2D, $\mathrm{D}^{\prime}$ ). These data are consistent with the hypothesis that a subset of Gb-Blimp-1-expressing cells receive and transduce BMP signals, and differentiate into PGCs during embryogenesis.

Gb-Blimp-1 is required for PGC formation and/or maintenance In order to test this hypothesis, we performed functional analysis using RNAi. We generated two non-overlapping double-stranded 
(ds) RNAs corresponding to either the SET or ZF domain of Gb-Blimp-1 (Fig. 1A); we refer to RNAi experiments performed with these sequences as Gb-Blimp-1 RNAi (SET) and Gb-Blimp-1 RNAi (ZF), respectively. As described below, both fragments showed statistically significant effects on PGCs (Fig. 3B-F, Fig. S4B,C, Figs S6-S8), and these phenotypic effects were not significantly different from each other (Fig. S4A, Figs S6-S8). We therefore used the Gb-Blimp-1 RNAi (SET) fragment for all subsequent analyses. We used quantitative PCR (qPCR) to confirm that RNAi resulted in significant reductions in transcript levels: the relative abundance of Gb-Blimp-1 mRNA was lowered to $48 \%$ in Gb-Blimp-1 RNAi embryos compared with buffer-injected control eggs (Fig. 3A).

Because knockdown of Prdm family members affects multiple developmental processes in other animals (reviewed by Hohenauer and Moore, 2012), we first asked whether Gb-Blimp-1 RNAi embryos showed any morphological abnormalities at 4 days AEL (ES8.5-9). We detected no defects in major embryonic patterning events, including axial patterning, segmentation and appendage elongation, nor did Gb-Blimp-1 RNAi cause significant developmental delays (data not shown). Because Gb-Blimp-1 expression was not completely reduced by our RNAi treatments (Fig. 3A), we cannot formally rule out a role for Gb-Blimp-1 in somatic patterning in G. bimaculatus. Nevertheless, we note that, similar to our cricket Gb-Blimp-1 knockdown embryos, Blimp-1 heterozygous knockout mice are healthy, normally patterned and fertile, but have a significantly reduced number of PGCs compared with wild-type control mice (Vincent et al., 2005). Therefore, we proceeded to quantify PGCs in Gb-Blimp-1 RNAi embryos.

We quantified PGCs in each embryonic abdominal segment by staining ES8.5-9 (4 days AEL) embryos with an anti-Gb-Piwi antibody as previously described (Donoughe et al., 2014); at earlier stages, PGCs are loosely scattered with variable Gb-Piwi expression levels and easily quantified PGC clusters have not yet formed (Ewen-Campen et al., 2013). Knockdown of Gb-Blimp-1 using either dsRNA fragment resulted in a significant reduction of PGCs. Specifically, Gb-Blimp-1 (SET) RNAi embryos showed a significant reduction of both PGC cluster size $(P<0.001, n=216$; Fig. 3D,F) and total PGC number per embryo $(P<0.001, n=18$; Fig. 3B, Fig. S4B) compared with controls. Similarly, $10.5 \%$ of Gb-Blimp-1 (ZF) RNAi embryos lacked PGCs altogether $(P=0.4176, n=19$; Table $S 2)$, and the remaining $89.5 \%$ of embryos showed a significant reduction of both PGC cluster size $(P<0.001, n=228$; Fig. S4C) and total PGC number per embryo $(P<0.001, n=19$; Fig. S4B) compared with control embryos. There was no significant difference in PGC cluster volume or PGC number between embryos injected with either of the two distinct dsRNA sequences (SET or ZF) used for Gb-Blimp-1 knockdown (Figs S6-S8), suggesting that the PGC phenotypes that we observed are not due to off-target effects.

\section{Gb-Blimp-1 is downstream of BMP signaling}

In both Gb-Blimp-1 RNAi treatments, we noted that not all segments of a given embryo were equally severely affected with respect to PGC number. Specifically, A2 and A3 were more strongly affected than A4 (Fig. 3F, Fig. S4D). This is consistent with our previous observations of segment-specific severity of PGC loss upon RNAi of BMP ligands (Donoughe et al., 2014). These observations, together with the known BMP dependence of Blimp-1 in mouse PGC formation (Ohinata et al., 2005), suggested that $G b$ Blimp-1 activity might be regulated by inputs from BMP signals to specify a subset of mesoderm as germ cells in each of abdominal segments A2-A4.
To test this hypothesis, we investigated the expression of Gb-Blimp1 in embryos with decreased or increased BMP levels, which we achieved by Gb-Mad RNAi or injection of recombinant Drosophila
A
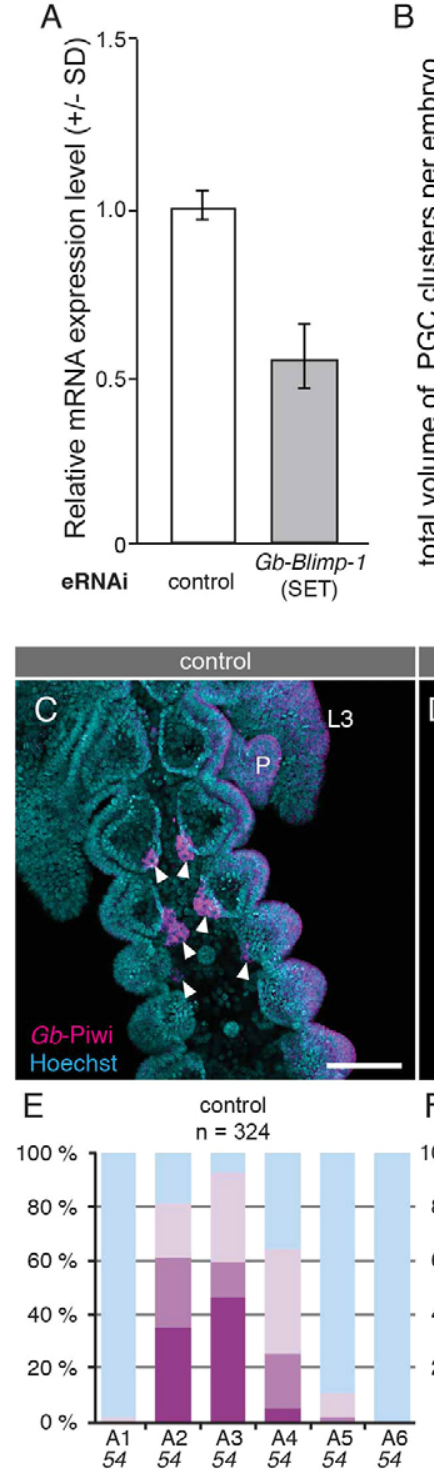

size class

medium: 5,000-10,000 $\mu^{3}{ }^{3}, 5-20$ PGCs
B

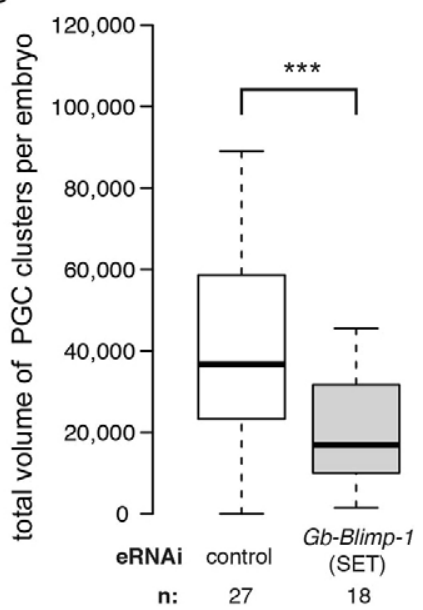

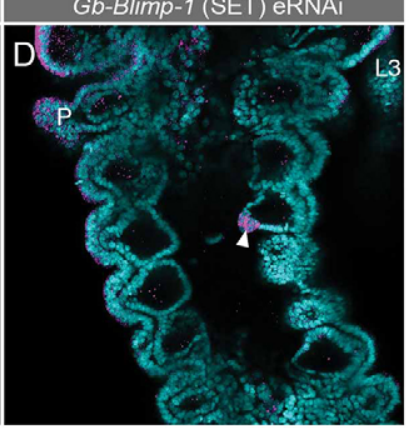

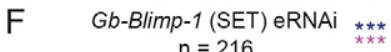

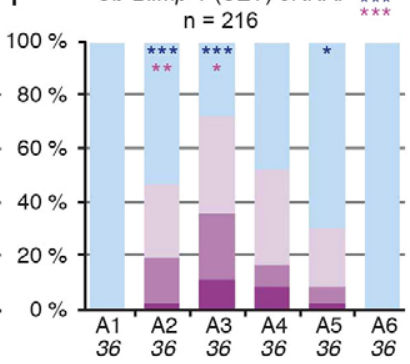

(1)

small: $<5,000 \mu \mathrm{m}^{3},<5$ PGCs

large: $>10,000 \mu \mathrm{m}^{3},>20$ PGCs

Fig. 3. Gb-Blimp-1 is required for PGC formation. RNAi against Gb-Blimp-1 results in loss of PGCs. (A) Gb-Blimp-1 (SET) RNAi applied at 0 day (0-5 h) AEL reduces transcript levels to $\sim 50 \%$ of control levels by 2.5 days. (B) The total volume of PGC clusters per embryo is significantly reduced in Gb-Blimp-1 (SET) RNAi embryos compared with controls. PGC cluster volume is positively correlated with PGC number (Donoughe et al., 2014). Thick black lines indicate the median; boxes indicate the interquartile range (IQR); whiskers extend to data points that are less than $1.5 \times$ the IQR away from first/third quartile. A MannWhitney test was used to calculate significance of comparison to control. $(C, D)$ Abdominal segments A1-A6 in a representative embryo from control (C) and Gb-Blimp-1 (SET) RNAi (D) treatments at 4 days AEL. PGC clusters are labeled with anti-Gb-Piwi antibody (magenta) and are indicated by arrowheads $P$, pleuropodia; $L 3$, third thoracic leg. (E,F) PGC quantification per segment at 4 days AEL for control (E) and Gb-Blimp-1 RNAi (F) embryos. Blue asterisks indicate significance of presence/absence of PGC clusters compared with controls; pink asterisks indicate significance of size differences of PGC clusters compared with controls. Mann-Whitney test was used to calculate significance in B,E,F. ${ }^{*} P<0.05,{ }^{* *} P<0.01,{ }^{* * *} P<0.001$. Scale bar: $100 \mu \mathrm{m}$ (C also applies to $\mathrm{D}$ ). 


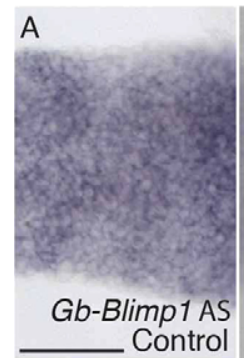

B

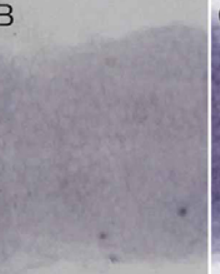

Gb-Blimp1 AS Control Gb-madRNAi
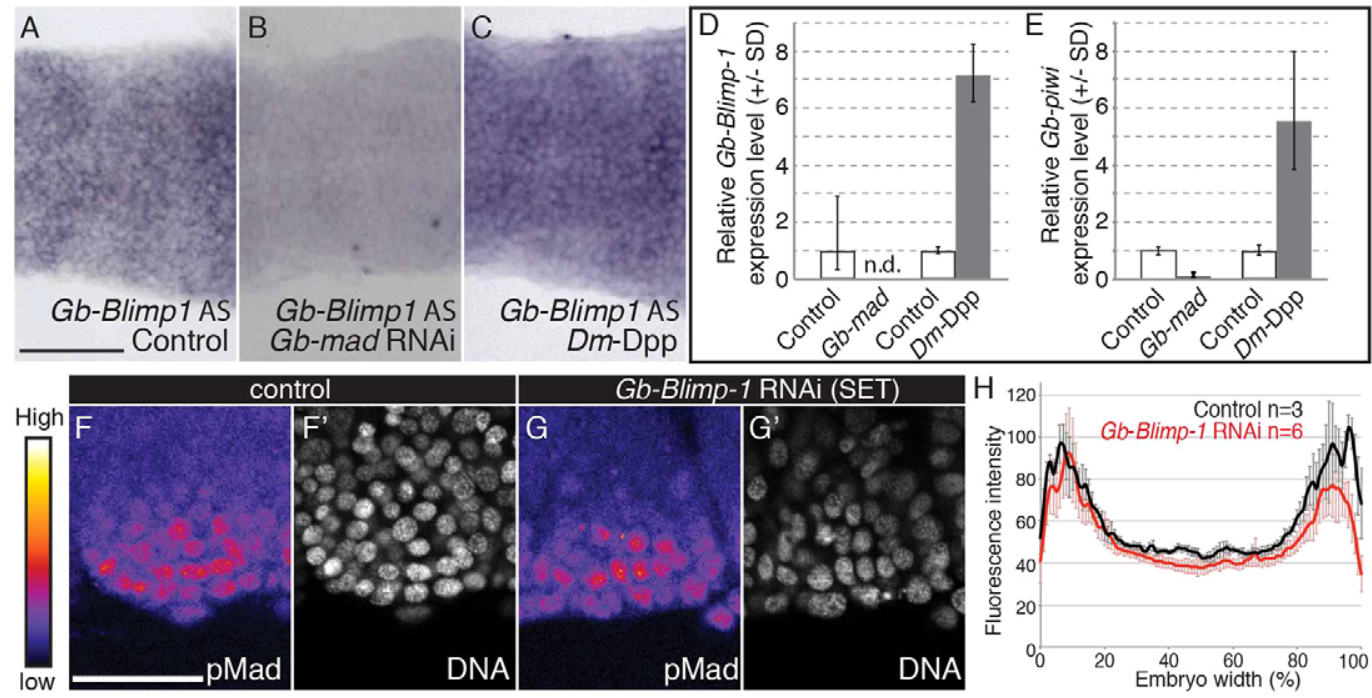

Fig. 4. Gb-Blimp-1 acts downstream of BMP signals to regulate PGC formation. (A-C) In situ hybridization for Gb-Blimp-1 in A2-A3 regions of control (A), GbMad RNAi (B) or recombinant Drosophila Dpp (Dm-Dpp) protein-injected embryos (C) at 2.5 days AEL suggest that BMP signaling positively regulates $G b-B l i m p-$ 1 expression. (D,E) qPCR validation of BMP signaling-mediated positive regulation of (D) Gb-Blimp-1 and (E) Gb-piwi transcript levels in Gb-Mad RNAi or DmDpp protein-injected embryos compared with controls. Bars show relative expression levels normalized to $G b$ - $\beta$-tubulin; biological triplicate data is presented as mean \pm s.d. n.d., not detected. ( $\left.F-G^{\prime}\right)$ pMad expression levels are shown with a rainbow heat map in the lateral A3 mesoderm at 2.5 days AEL, where PGCs arise, in representative control and Gb-Blimp-1 (SET) RNAi embryos. (H) Quantified average intensity profiles of pMad levels in the mesodermal region of each of segments A2-A4 of controls (black) and RNAi (red) embryos shows that reduction of Gb-Blimp-1 levels does not alter nuclear pMad levels. Error bars in $\mathrm{H}$ represent s.d. Scale bars: $100 \mu \mathrm{m}$.

Dpp protein (Dm-Dpp), respectively (Fig. 4). We previously showed that Gb-Mad RNAi decreases BMP signaling levels, leading to a reduction of nuclear pMad accumulation and loss or significant reduction of PGCs, and, conversely, that Dm-Dpp injection elevates BMP signaling levels, leading to an increase in nuclear pMad accumulation and a dose-dependent increase of PGC cluster size in every segment (Donoughe et al., 2014). Focusing on ES6, the stage when PGCs first arise, we used in situ hybridization to characterize qualitative changes in Gb-Blimp-1 expression in treated embryos, and qPCR to quantify the expression levels of Gb-Blimp-1 and Gb-piwi transcripts in abdominal segments A2-A4. In control embryos, we observed the same $G b$-Blimp-1 expression pattern as that described for wild-type embryos (Fig. 1), namely, low levels of uniform expression across the whole embryo, with domains of higher expression in A2-A4 where PGCs arise (Fig. 4A). In contrast to control embryos, Gb-Mad RNAi embryos lacked both the uniformly and segmentally enriched Gb-Blimp-1 expression patterns (Fig. 4B). Conversely, in Dm-Dpp protein-injected embryos, Gb-Blimp-1 expression was increased uniformly across the embryo, with no apparent enrichment in A2-A4 (Fig. 4C). These in situ hybridization observations were validated by qPCR: Gb-Blimp-1 expression was undetectable in A2A4 of Gb-Mad RNAi embryos, but was 7.1 \pm 0.1 -fold higher in A2-A4 of Dm-Dpp-injected embryos than in controls (Fig. 4D). In addition, reduced or elevated levels of BMP signaling caused corresponding changes not only in Gb-Blimp-1 transcript levels, but also in Gb-piwi transcript levels in A2-A4 (Fig. 4E). Taken together, these results suggest that, as in mice (Ohinata et al., 2009), G. bimaculatus PGC fate (as revealed by Gb-piwi expression) could be regulated by BMPdependent Gb-Blimp-1 activity. Our data do not allow us to determine whether activation of Gb-Blimp-1 by BMP signaling, or activation of Gb-piwi by Gb-Blimp-1, is direct or indirect. We note that, similarly, whether Blimp-1 regulates Piwi-like transcription directly or indirectly is also currently unknown in mice (Saitou and Yamaji, 2012).

We next asked whether the regulatory relationship between BMP signaling and Blimp-1 is reciprocal, by assessing the levels of BMP signaling in a Gb-Blimp- 1 knockdown background. pMad activity in the dorsolateral regions of ectoderm and mesoderm tissues was unaffected in Gb-Blimp-1 RNAi embryos at ES6 (Fig. 4F-H). Furthermore, the loss of PGCs in Gb-Blimp-1 RNAi embryos is unlikely to be due to changes in proliferation or apoptosis rates of mesodermal cells or PGCs, as we observed no significant differences in these parameters in Blimp-1 RNAi embryos compared with controls (Fig. S9). Taken together, these data indicate that at the time and place of cricket PGC specification, Gb-Blimp-1 acts downstream of BMP signals to specify the PGCs but does not regulate BMP signaling in a positive- or negative-feedback loop.

To further examine the functional relationship between the $G b$ Blimp-1 and BMP signals that induce PGC specification, we performed double-RNAi experiments against Gb-Blimp-1 and each of the two most relevant BMP ligands for this process (Donoughe et al., 2014), namely $G b-d p p l$ and $G b-g b b$, and the downstream effector $G b-M a d$, and quantified PGCs at 4 days AEL in these double-RNAi embryos (Fig. 5, Figs S5-S8). We previously found that if RNAi was induced $0-5 \mathrm{~h}$ ( 0 day) AEL, many BMP pathway RNAi embryos displayed severe morphological defects suggestive of dorsalization (Donoughe et al., 2014), consistent with a conserved role for BMP signaling in dorsoventral patterning (Niehrs, 2010). In order to bypass this early requirement for BMP signaling in axial patterning, we injected a mixture of Gb-Blimp-1 and BMP pathway gene dsRNAs into ES2-4 embryos (30-36 h AEL, hereafter referred to as 1.5 days AEL), which have completed initial axial patterning but not yet specified PGCs, and quantified PGCs in the resulting double-RNAi embryos that displayed wildtype gross morphology. We then compared these data with PGC quantifications from parallel single-RNAi experiments for $G b$ Blimp-1 and each tested BMP pathway gene (Fig. 5, Fig. S5-S8).

A total of $20.7 \%$ of Gb-Blimp-1 RNAi (1.5 days AEL) embryos lacked PGCs, and the remaining $79.3 \%$ had significantly smaller PGC clusters $(P<0.001, n=348$; Fig. S5I,J) and significantly fewer PGCs than buffer-injected controls $(P<0.001, n=29$; Fig. 5). There 


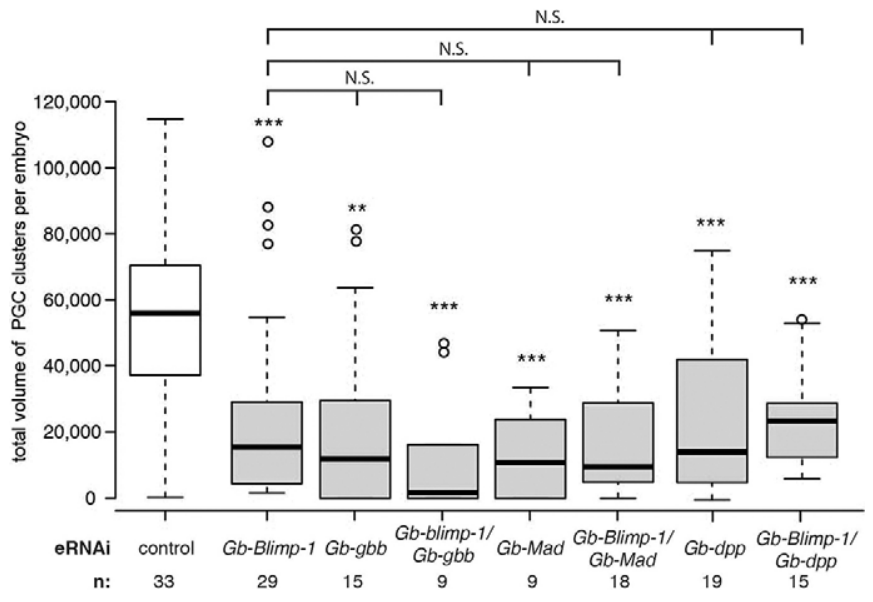

Fig. 5. The effect of double knockdowns of Gb-Blimp-1 (SET) and BMP signaling pathway genes on total volume of PGC clusters. Box-whisker plots showing the distribution of total volumes of PGC clusters in segments A1 to A6 per embryo, including absent clusters (clusters with volume of $0 \mu \mathrm{m}^{3}$ ) compared with control embryos. Thick black lines, median; boxes, interquartile range (IQR); whiskers extend to data points that are less than $1.5 \times$ the IQR from first/third quartile. A Mann-Whitney test was used to calculate the significance of comparison with controls, and a Steel-Dwass test was used to compare values among RNAi-treated embryos. N.S., not significant; ${ }^{* *} P<0.01$, ${ }^{* * *} P<0.001$

were no significant differences in total PGC cluster size between embryos where Gb-Blimp-1 RNAi was performed at 0 day AEL or 1.5 days AEL $(P<0.179$; Figs $\mathrm{S} 6$ and S7). These data are, like the 0 day AEL RNAi experiments described above, consistent with a role for Gb-Blimp-1 in PGC specification and/or maintenance.

Single knockdowns of BMP pathway members significantly reduced PGC cluster size ( $G b-M a d$ RNAi, $P<0.001, n=108$; $G b-g b b$ RNAi, $P<0.001, n=180$; Gb-dpp 1 RNAi, $P<0.001, n=222$; Fig. S6) and total PGC number per embryo (Gb-Mad RNAi, $P<0.001, n=9$; $G b-g b b$ RNAi, $P<0.01, n=15$; Gb-dpp 1 RNAi, $P<0.001, n=19$; Fig. 5), consistent with previous reports (Donoughe et al., 2014). Double knockdowns of $G b-d p p 1$ or $G b-M a d$ together with $G b$ Blimp-1 RNAi had significantly fewer PGCs than buffer-injected controls, but were not phenotypically different from the single knockdowns of any of these genes by any measurement, including axial patterning, mesoderm formation, overall morphology, or PGC number or distribution (Fig. 5, Figs S6 and S7).

Our analysis of the double knockdown of $G b$-gbb and Gb-Blimp1 yielded results suggesting segment-specific requirements for BMP-Gb-Blimp-1 interactions. These embryos were morphologically wild type (Fig. S5A-D). Those embryos that possessed PGCs had significantly smaller PGC clusters $(P<0.001$, $n=108$; Fig. S5I,L) and significantly fewer total PGCs $(P<0.001$, $n=9$; Fig. 5) than buffer-injected controls, although comparing the total PGC number per embryo between double-knockdown and single-knockdown embryos showed no differences (Steel-Dwass test, Fig. 5). However, when we examined the distribution of PGC clusters, we found that the A4 segment (but not A2 or A3) of $G b$ gbb/Gb-Blimp-1 double-knockdown embryos completely lacked PGCs significantly more frequently than in the Gb-Blimp-1 singleknockdown condition $(P<0.01, n=54$; Fig. S8C,D). By contrast, the proportion of A4 segments completely lacking PGCs was not significantly affected by Gb-Blimp-1 RNAi alone, whether performed at 0 day or 1.5 days AEL, compared with bufferinjected controls (Fig. S8C). In other words, while PGC formation in all segments A2-A4 was hindered by single knockdown of either
$G b$-gbb or Gb-Blimp-1, knocking down both genes together caused even more severe PGC loss in A4, but not in A2 or A3. This suggests that the requirement for, or functions of, Gb-Blimp-1 and $G b-g b b$ in $G$. bimaculatus PGC formation may differ between abdominal segments. Specifically, we speculate that, although the essential molecular mechanisms for specifying PGCs are the same across the A2-A4 segments, different segments might have different levels of sensitivity to the relevant signals.

\section{DISCUSSION \\ Conservation and divergence of somatic Blimp-1 functions across animals}

Although our expression and functional analyses of Gb-Blimp-1 point to a role in G. bimaculatus PGC formation, its expression pattern suggests that it might have somatic functions as well. For example, we noticed transiently enriched expression of Gb-Blimp-1 transcripts in each newly formed abdominal segment during the posterior elongation processes. In D. melanogaster, the Blimp-1 ortholog also exhibits some segmental expression: Drosophila Blimp-1 is expressed progressively as four striped patterns along the anteroposterior (AP) axis at blastoderm stages. These striped patterns are gap gene-like: that is, they are wide enough to suggest that they encompass multiple segments, and the expression is transient, disappearing as gastrulation proceeds ( $\mathrm{Ng}$ et al., 2006). Despite these suggestive expression patterns, however, loss of Blimp-1 does not yield embryonic segmentation defects in Drosophila (Ng et al., 2006). Similarly, in G. bimaculatus we detected no segmental defects in relatively late stage (ES8-9) $G b$ Blimp-1 RNAi embryos. However, we would not have detected such embryos in our analysis, as we selected only those embryos with intact segmentation for PGC quantification in order to eliminate 'false positive' PGC loss that could result from defects in axial elongation or segment generation. We therefore consider it formally possible that Gb-Blimp-1 plays some role in early segment identity or generation.

In terms of its expression and function in other animals, a common feature of somatic Blimp-1 expression appears to be its general restriction to the anterior inner germ layers (mesoderm and/or endoderm). In some cases its expression appears coincident with PGC origin, in the somatic tissue that gives rise to PGCs. Here, we have reported expression of Gb-Blimp-1 in the abdominal mesoderm of PGC-originating segments prior to, during and following PGC specification in $G$. bimaculatus. Other studies report Blimp-1 expression suggestive of a PGC role, but the data are complex. For example, Blimp- 1 is expressed in the sea star endomesoderm, which is the tissue that gives rise to the posterior enterocoel (PE), a structure thought to be the source of the germ cells in this animal (Fresques et al., 2014). Sea star Blimp-1 endomesodermal expression begins before the formation of the PE, becoming enriched in the midgut region where the PE will form. However, following PE formation, while expression of the germ line marker genes vasa and piwi becomes enriched in the PE, suggesting the onset of PGC specification, Blimp-1 expression levels are not detected in that region. This suggests that sea star Blimp-1 might play a general role in $\mathrm{PE}$ formation or maintenance of gut pluripotency, rather than in germ cell segregation (Fresques et al., 2014).

Within vertebrates that specify PGCs by induction, such as in rabbit and mouse, Blimp-1 is expressed in individual mesodermal cells where PGCs arise (Vincent et al., 2005; Hopf et al., 2011), and the role of Blimp-1 in mouse PGC specification is well established (Ohinata et al., 2005). However, mouse Blimp-1 is also expressed in a number of anterior inner layer-derived tissues, including the axial mesendoderm, 
which provides anterior patterning signals, and the branchial arches (de Souza et al., 1999). Although Blimp-1 knockout mice show wildtype axial patterning overall, they also exhibit loss of caudal branchial arches and loss of PGCs (Vincent et al., 2005; Robertson et al., 2007). In contrast to the role of mouse Blimp-1, in axolotls, which also use BMP-based inductive signaling to specify PGCs, Blimp-1 transcripts are not expressed in the lateral mesoderm where PGCs arise (Chatfield et al., 2014). To our knowledge, the function of axolotl Blimp-1 has not been directly examined, but given its expression pattern it seems unlikely to play a role in PGC specification. In the lamprey, in which late embryonic PGC specification suggests an inductive mechanism (Beard, 1902; Okkelberg, 1921; Smith et al., 2012), Blimp-1 is expressed in various inner layer embryonic tissues including the anterior mesendoderm, premigratory neural crest and branchial arches, and loss of Blimp-1 leads to incorrect positioning of the AP axis (Nikitina et al., 2011).

In vertebrates that specify PGCs using maternally derived determinants, Blimp-1 nevertheless plays a number of somatic roles, which can again be broadly described as anterior patterning of inner layer tissues. In $X$. laevis, Blimp-1 transcripts are found in the anterior endomesoderm (reminiscent of its expression in the analogous mouse tissue, the anterior visceral endoderm). In $X$. laevis embryos Blimp-1 represses brachyury, Myf5 and chordin, and Blimp-1 overexpression leads to axial truncation. In X. laevis animal cap assays, Blimp-1 is capable of activating the anterior mesendoderm markers cerberus and goosecoid (de Souza et al., 1999). Similarly, in the zebrafish D. rerio, the Blimp-1 homolog $u$-boot (ubo, prdm1a) is expressed in the prechordal mesoderm and mesendoderm at $70 \%$ epiboly. ubo morphants show truncation of the body axis and loss of the head primordium (Baxendale et al., 2004), as well as branchial arch defects or loss (Wilm and SolnicaKrezel, 2005). Taken together, these data indicate that although Blimp-1 orthologs play diverse roles in the development of extant animals, ancestral features of vertebrate Blimp-1 function are likely to have included roles in anterior inner layer formation, maintenance and/or patterning. According to this hypothesis, Blimp-1 would have lost its role in axial patterning and gained a role in PGC specification in the lineage leading to mice, or potentially to mammals.

In mice, Blimp-1 promotes PGC fate in part by inhibiting somatic fate in presumptive PGCs (Ohinata et al., 2005). Given the widespread mesodermal expression of Gb-Blimp-1, it is possible that an analogous mechanism operates in the cricket. The most relevant data we have at the moment to address this question comes from our analysis of apoptosis and proliferation among mesodermal cells in Gb-Blimp-1 RNAi embryos: both of these behaviors appear unchanged compared with controls (Fig. S9). Although the number of mesodermal cells appears to remain constant in Gb-Blimp-1 RNAi embryos, it is possible that some cell fate changes have occurred among these cells. If this is the case, however, these cell fate changes do not appear to have significant impacts on abdominal development, as we observe normal overall mesodermal behaviors and coelomic pouch morphologies in Gb-Blimp-1 RNAi embryos. At present, no molecular markers are available that reveal distinct fates of subsets of mesodermal cells in the developing cricket abdomen, hence our reliance herein on assessments of proliferation, apoptosis and morphology to determine if cell fates have been correctly allocated. Even if all of the mesodermal cells that would normally have given rise to PGCs adopted somatic fate in $G b$ Blimp-1 RNAi embryos, this would be unlikely to add more than $\sim 50$ cells to the mesodermal pool in segments A2-A4. Unlike in mouse Blimp- 1 heterozygotes, we do not observe a distinct cluster of putative misspecified PGCs in Gb-Blimp-1 RNAi embryos.

\section{Upstream regulation of Blimp-1}

Despite the diverse developmental roles played by Blimp-1 in different animals, a widely shared feature of Blimp-1 function is its regulation by the BMP signaling pathway. This is true whether Blimp-1 operates in the soma or in the germ line. BMP signals positively regulate Blimp-1 expression in the context of PGC specification in mice (Ohinata et al., 2005) and cricket (Donoughe et al., 2014; this study). In zebrafish, $u b o$ expression at the neural plate border is induced by BMP signaling (Roy and Ng, 2004). Taken together, these data suggest that in these vertebrates and invertebrates a positive regulatory relationship between BMP signaling and Blimp-1 expression is conserved. However, the specific molecular mechanisms that mediate BMP induction of Blimp-1 appear to vary across animals. In mice, Blimp-1 activation during PGC specification proceeds through brachyury $(T)$, which directly activates Blimp- 1 under conditions of active BMP signaling, but is repressed in the absence of BMP signals (Aramaki et al., 2013). However, in the case of axolotl PGC specification, Blimp-1 is not expressed in the lateral mesoderm, which is where PGCs arise, and BMP signals cannot activate Blimp-1 expression in animal cap assays (Chatfield et al., 2014). Axolotl PGCs are of mesodermal origin, and their fate is induced by the combinatorial functions of brachyury, BMP and FGF signaling, but brachyury and Bmp4 signals cannot induce Blimp-1 expression in the absence of FGF signaling (Chatfield et al., 2014). In G. bimaculatus, Gb-brachyury transcripts are detected in the posterior growth zone and in the developing hindgut through embryogenesis but not in the mesoderm of any body segment (Shinmyo et al., 2006). Thus, Gb-Blimp-1 transcripts are unlikely to be co-expressed with Gb-brachyury in abdominal segments A2-A4 during the PGC induction stage. Furthermore, knockdown analysis by RNAi revealed that $G b$ brachyury is not required for AP axial elongation or normal segment formation, but is essential for posterior gut formation (Shinmyo et al., 2006). It is thus unlikely that $G b$-brachyury regulates $G b$ Blimp-1 expression in the context of $G$. bimaculatus PGC specification. However, we cannot formally rule out the possibility that the presumptive abdominal mesoderm utilizes or requires brachyury signals from the growth zone at a stage prior to the formation of abdominal segments A2-A4 and PGC specification in those segments.

\section{Gb-Blimp-1 may play segment-specific roles in PGC specification and development}

Double knockdowns of Gb-Blimp-1 and BMP pathway members suggested that PGC specification and/or maintenance is more sensitive to a simultaneous reduction of both $G b-g b b$ and $G b$ Blimp-1 in A4 than in A2 or A3. However, this result cannot be explained by a greater requirement for each gene individually in this segment, since single-knockdown embryos for either gene showed a greater reduction in PGC number in A2 and A3, rather than in A4 (Fig. 3F, Fig. S5J) (Donoughe et al., 2014). This was also the case for a second BMP ligand, Gb-dppl (Fig. S5O), whereas single knockdown of the downstream effector $G b$-Mad appeared to affect all segments equally (Fig. S5M). Similarly, elevated levels of BMP signaling result in supernumerary and ectopic PGCs, but these do not appear with equal frequency in all segments (Donoughe et al., 2014). Taken together, these data support our previous proposal (Donoughe et al., 2014) that PGCs are differentially sensitive to BMP signaling along the AP axis. In light of the data shown in this study, and the wellestablished conserved role of Hox genes in conferring positional identity along the anterior-posterior axis of animals, we speculate that combinatorial positional information provided by BMP pathway 
activity, Blimp-1 activity, and Hox genes regulates the formation of PGCs in the correct abdominal segments of $G$. bimaculatus.

\section{Evolutionary hypotheses regarding the role of Blimp-1 in PGC specification}

Blimp-1 is associated with PGCs in a number of vertebrates: it is required downstream of BMP signaling for PGC specification in mice (Ohinata et al., 2005), and spatiotemporal expression patterns of Bmp 2/4 and/or Blimp-1 are associated with the distribution of PGCs in rabbit and chicken (Hopf et al., 2011; Wan et al., 2014), although chicken (Tsunekawa et al., 2000), unlike mammals (reviewed by Extavour, 2007), may use a germ plasm-based PGC specification mechanism. Here we present, for the first time, evidence that Blimp-1 is also required for the specification of PGCs in a protostome (Fig. 6). The Blimp-1-based mechanism of germ cell induction in $G$. bimaculatus is similar to that observed in mouse: in both cases, Blimp-1 expression is induced in the abdominal mesoderm by BMP signaling and is essential for the generation of PGCs. This mechanism could have evolved independently in the lineages leading to mammals (or amniotes) and insects, which would be consistent with the absence of a function for Blimp-1 in axolotl PGC induction (Chatfield et al., 2014). However, given that Blimp-1 and BMP signaling components clearly predate bilaterian radiation, and that Blimp-1-BMP interactions are widely conserved in both germ cell and somatic development, we propose that the accumulated evidence to date most strongly support the hypothesis that $\mathrm{BMP} /$ Blimp-1-based PGC induction was present in a last common bilaterian ancestor. According to this hypothesis, the BMP/Blimp-1 PGC specification mechanism would have been subsequently lost in lineages that evolved inheritance (germ plasm)-based PGC specification mechanisms. In lineages such as axolotl, BMP signaling would have been retained to induce germ cells but variation evolved in the mechanisms cooperating with, and operating downstream of, BMP signaling such that the role of Blimp-1 in this process became dispensable. Future studies investigating the role of Blimp-1 in a wider range of protostomes, and wider taxon sampling to determine the extent of diversity of BMP-associated molecular mechanisms that operate in PGC specification, will be required to test these hypotheses.

\section{MATERIALS AND METHODS}

\section{Animals}

G. bimaculatus were reared under standard conditions as previously described (Kainz et al., 2011)

\section{Cloning and RNAi for the Gryllus homolog of Blimp-1}

A Gryllus homolog of Blimp-1 was cloned using sequences from the Gryllus developmental transcriptome (Zeng et al., 2013) and from an unpublished Gryllus brain transcriptome (T. Bando, Okayama University, Japan, personal communication) by PCR using cDNA from whole embryos at 4 days AEL. The cDNA sequence has been deposited in GenBank (accession number KR861513). Preparation of dsRNA and embryonic RNAi were performed as previously described (Ewen-Campen et al., 2013).

\section{Whole-mount in situ hybridization and immunohistochemistry}

Whole-mount in situ hybridization was performed as described (Kainz et al., 2011). Antibody staining was carried out according to standard protocols (Patel, 1994). Double detections of transcripts and proteins were carried out as previously described (Donoughe et al., 2014). For further information, including details of the antibodies used, see the supplementary materials and methods.

\section{Quantitative PCR (qPCR)}

Anterior abdominal segments A1-A5 were dissected from control or $G b$ Blimp-1 RNAi-treated embryos ( $n=10$ per treatment) using fine tungsten needles, and segments from ten embryos were pooled into single tubes.

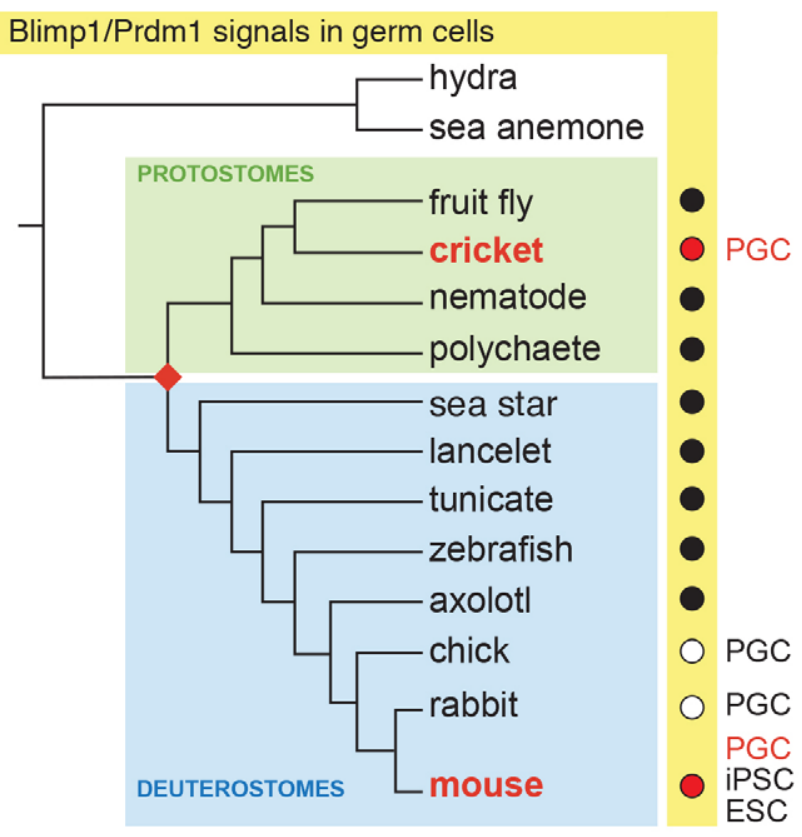

Fig. 6. Blimp-1 (Prdm1) signals in germ cells across the Metazoa. Phylogenetic distribution of selected animal taxa, indicating evidence for Blimp-1 function in the context of germ cell development [see Donoughe et al. (2014)], based either on expression data (white circles) or functional data (red circles). Black circles indicate that expression and/or functional data suggest that Blimp-1 is not involved in germ line development. Red text indicates taxa for which functional data support a role for BMP-based Blimp-1 signaling in PCG specification. A red diamond represents the hypothesis that PGC induction by BMP-based Blimp-1 activity is ancestral in Bilateria. ESC, conversion of embryonic stem cells to germ cells; iPSC, conversion of induced pluripotent stem cells to germ cells; PGCs, primordial germ cells.

Total RNA was extracted using Trizol (Life Technologies) following the manufacturer's directions. RNA pools were divided into two samples and each half was reverse transcribed to prepare cDNA using SuperScript III (Invitrogen). A no-reverse transcriptase control was performed in parallel for each sample. Each cDNA was divided into three samples and used for qPCR. An MxP3005 machine (Stratagene) was used for qPCR as previously described (Donoughe et al., 2014). Relative transcript ratios in the qPCR study were calculated from experiments performed in triplicate and are shown as mean \pm s.d. in Fig. 3A and Fig. S4A. The housekeeping gene Gryllus $\beta$-tubulin was used as an internal control as previously described (Donoughe et al., 2014). Primers are listed in Table S1.

\section{BMP pathway activation}

BMP activation in G. bimaculatus embryos was achieved by injecting recombinant $D$. melanogaster Dpp protein as described in the supplementary materials and methods.

\section{Quantification of PGCs}

Quantification of PGCs was conducted as previously described (Donoughe et al., 2014).

\section{Statistical analysis}

For comparison of two independent samples, non-normally distributed variables were compared by the Mann-Whitney U-test. The Steel-Dwass test, which is a non-parametric post-hoc test, was used for all pairwise comparisons of results from three or more groups.

\section{Acknowledgements}

We thank members of the C.G.E. laboratory for discussions and comments on the manuscript.

\section{Competing interests}

The authors declare no competing or financial interests. 


\section{Author contributions}

T.N. performed all experiments, collected and analyzed data. T.N. and C.G.E. designed the research, prepared the figures and wrote the paper.

\section{Funding}

This research was supported by a National Science Foundation grant [IOS1257217] to C.G.E. and a Japan Society for the Promotion of Science (JSPS)

Postdoctoral Fellowship for Research Abroad to T.N

\section{Supplementary information}

Supplementary information available online at

http://dev.biologists.org/lookup/suppl/doi:10.1242/dev.127563/-/DC1

\section{References}

Agawa, Y., Sarhan, M., Kageyama, Y., Agaki, K., Takai, M., Hashiyama, K. Wada, T., Handa, H., Iwamatsu, A., Hirose, S. et al. (2007). Drosophila Blimp-1 is a transient transcriptional repressor that controls timing of the ecdysoneinduced developmental pathway. Mol. Cell. Biol. 27, 8739-8747.

Akagi, K. and Ueda, H. (2011). Regulatory mechanisms of ecdysone-inducible Blimp-1 encoding a transcriptional repressor that is important for the prepupal development in Drosophila. Dev. Growth Differ. 53, 697-703.

Aramaki, S., Hayashi, K., Kurimoto, K., Ohta, H., Yabuta, Y., Iwanari, H., Mochizuki, Y., Hamakubo, T., Kato, Y., Shirahige, K. et al. (2013). A mesodermal factor, $\mathrm{T}$, specifies mouse germ cell fate by directly activating germline determinants. Dev. Cell 27, 516-529.

Baxendale, S., Davison, C., Muxworthy, C., Wolff, C., Ingham, P. W. and Roy, S. (2004). The B-cell maturation factor Blimp-1 specifies vertebrate slow-twitch muscle fiber identity in response to Hedgehog signaling. Nat. Genet. 36, 88-93.

Beard, J. (1902). The germ cells of Pristiurus. Anat. Anz. 21, 50-61.

Chatfield, J., O’Reilly, M.-A., Bachvarova, R. F., Ferjentsik, Z., Redwood, C. Walmsley, M., Patient, R., Loose, M. and Johnson, A. D. (2014). Stochastic specification of primordial germ cells from mesoderm precursors in axolot embryos. Development 141, 2429-2440.

de Souza, F. S., Gawantka, V., Gomez, A. P., Delius, H., Ang, S. L. and Niehrs, C. (1999). The zinc finger gene Xblimp1 controls anterior endomesodermal cell fate in Spemann's organizer. EMBO J. 18, 6062-6072.

Donoughe, S. and Extavour, C. G. (2015). Embryonic development of the cricke Gryllus bimaculatus. Dev. Biol. pii: S0012-1606(15)00189-X

Donoughe, S., Nakamura, T., Ewen-Campen, B., Green, A. D., II, Henderson, L. and Extavour, C. G. (2014). BMP signaling is required for the generation of primordial germ cells in an insect. Proc. Natl. Acad. Sci. USA 111, 4133-4138.

Ewen-Campen, B., Donoughe, S., Clarke, D. N. and Extavour, C. G. (2013) Germ cell specification requires zygotic mechanisms rather than germ plasm in a basally branching insect. Curr. Biol. 23, 835-842.

Extavour, C. G. (2007). Evolution of the bilaterian germ line: lineage origin and modulation of specification mechanisms. Integr. Comp. Biol. 47, 770-785.

Extavour, C. G. and Akam, M. E. (2003). Mechanisms of germ cell specification across the metazoans: epigenesis and preformation. Development 130 5869-5884.

Fresques, T., Zazueta-Novoa, V., Reich, A. and Wessel, G. M. (2014). Selective accumulation of germ-line associated gene products in early development of the sea star and distinct differences from germ-line development in the sea urchin. Dev. Dyn. 243, 568-587.

Hinman, V. F. and Davidson, E. H. (2003). Expression of AmKrox, a starfish ortholog of a sea urchin transcription factor essential for endomesodermal specification. Gene Expr. Patterns 3, 423-426.

Hohenauer, T. and Moore, A. W. (2012). The Prdm family: expanding roles in stem cells and development. Development 139, 2267-2282.

Hopf, C., Viebahn, C. and Püschel, B. (2011). BMP signals and the transcriptional repressor BLIMP1 during germline segregation in the mammalian embryo. Dev. Genes Evol. 221, 209-223.

Kainz, F., Ewen-Campen, B., Akam, M. and Extavour, C. G. (2011). Notch/Delta signalling is not required for segment generation in the basally branching insect Gryllus bimaculatus. Development 138, 5015-5026.

Lawson, K. A., Dunn, N. R., Roelen, B. A. J., Zeinstra, L. M., Davis, A. M., Wright, C. V. E., Korving, J. P. W. F. M. and Hogan, B. L. M. (1999). Bmp4 is required for the generation of primordial germ cells in the mouse embryo. Genes Dev. 13, 424-436.

Livi, C. B. and Davidson, E. H. (2006). Expression and function of blimp1/krox, an alternatively transcribed regulatory gene of the sea urchin endomesoderm network. Dev. Biol. 293, 513-525.

Mito, T. and Noji, S. (2009). The two-spotted cricket Gryllus bimaculatus: an emerging model for developmental and regeneration studies. In Emerging Model Organisms: A Laboratory Manual, pp. 331-346. Cold Spring Harbor: Cold Spring Harbor Laboratory Press.
Mito, T., Nakamura, T. and Noji, S. (2010). Evolution of insect development: to the hemimetabolous paradigm. Curr. Opin. Genet. Dev. 20, 355-361.

Ng, T., Yu, F. and Roy, S. (2006). A homologue of the vertebrate SET domain and zinc finger protein Blimp-1 regulates terminal differentiation of the tracheal system in the Drosophila embryo. Dev. Genes Evol. 216, 243-252.

Niehrs, C. (2010). On growth and form: a Cartesian coordinate system of Wnt and BMP signaling specifies bilaterian body axes. Development 137, 845-857.

Nieuwkoop, P. D. (1951). Experimental investigations on the origin and determination of the germ cells, and on the development of the lateral plates and germ ridges in urodeles. Arch. Néerland. de Zool. 8, 1-205

Nikitina, N., Tong, L. and Bronner, M. E. (2011). Ancestral network module regulating prdm1 expression in the lamprey neural plate border. Dev. Dyn. 240 2265-2271.

Ohinata, Y., Payer, B., O’Carroll, D., Ancelin, K., Ono, Y., Sano, M., Barton, S. C., Obukhanych, T., Nussenzweig, M., Tarakhovsky, A. et al. (2005). Blimp1 is a critical determinant of the germ cell lineage in mice. Nature 436, 207-213.

Ohinata, Y., Ohta, H., Shigeta, M., Yamanaka, K., Wakayama, T. and Saitou, M. (2009). A signaling principle for the specification of the germ cell lineage in mice. Cell 137, 571-584.

Okkelberg, P. (1921). The early history of the germ cells in the brook lamprey, Entosphenus wilderi (gage), up to and including the period of sex differentiation J. Morphol. 35, 1-151.

Patel, N. H. (1994). Imaging neuronal subsets and other cell types in whole-mount Drosophila embryos and larvae using antibody probes. Meth. Cell Biol. 44 445-487.

Robertson, E. J., Charatsi, I., Joyner, C. J., Koonce, C. H., Morgan, M., Islam, A., Paterson, C., Lejsek, E., Arnold, S. J., Kallies, A. et al. (2007). Blimp1 regulates development of the posterior forelimb, caudal pharyngeal arches, heart and sensory vibrissae in mice. Development 134, 4335-4345.

Roy, S. and Ng, T. (2004). Blimp-1 specifies neural crest and sensory neuron progenitors in the zebrafish embryo. Curr. Biol. 14, 1772-1777.

Saitou, M. and Yamaji, M. (2012). Primordial germ cells in mice. Cold Spring Harb. Perspect. Biol. 4, a008375.

Saitou, M., Barton, S. C. and Surani, M. A. (2002). A molecular programme for the specification of germ cell fate in mice. Nature 418, 293-300

Seetharam, A. and Stuart, G. W. (2013). A study on the distribution of 37 well conserved families of $\mathrm{C} 2 \mathrm{H} 2$ zinc finger genes in eukaryotes. BMC Genomics 14 420

Seki, Y., Yamaji, M., Yabuta, Y., Sano, M., Shigeta, M., Matsui, Y., Saga, Y. Tachibana, M., Shinkai, Y. and Saitou, M. (2007). Cellular dynamics associated with the genome-wide epigenetic reprogramming in migrating primordial germ cells in mice. Development 134, 2627-2638.

Shinmyo, Y., Mito, T., Uda, T., Nakamura, T., Miyawaki, K., Ohuchi, H. and Noji, S. (2006). brachyenteron is necessary for morphogenesis of the posterior gut but not for anteroposterior axial elongation from the posterior growth zone in the intermediate-germband cricket Gryllus bimaculatus. Development 133 4539-4547.

Smith, J. J., Baker, C., Eichler, E. E. and Amemiya, C. T. (2012). Genetic consequences of programmed genome rearrangement. Curr. Biol. 22 1524-1529.

Tsunekawa, N., Naito, M., Sakai, Y., Nishida, T. and Noce, T. (2000). Isolation of chicken vasa homolog gene and tracing the origin of primordial germ cells. Development 127, 2741-2750.

Turner, C. A., Mack, D. H. and Davis, M. M. (1994). Blimp-1, a novel zinc fingercontaining protein that can drive the maturation of B lymphocytes into immunoglobulin-secreting cells. Cell 77, 297-306.

Vincent, S. D., Dunn, N. R., Sciammas, R., Shapiro-Shalef, M., Davis, M. M., Calame, K., Bikoff, E. K. and Robertson, E. J. (2005). The zinc finge transcriptional repressor Blimp1/Prdm1 is dispensable for early axis formation but is required for specification of primordial germ cells in the mouse. Development $132,1315-1325$

Wan, Z., Rui, L. and Li, Z. (2014). Expression patterns of prdm1 during chicken embryonic and germline development. Cell Tissue Res. 356, 341-356.

Wang, W., Wikramanayake, A. H., Gonzalez-Rimbau, M., Vlahou, A., Flytzanis, C. N. and Klein, W. H. (1996). Very early and transient vegetal-plate expression of SpKrox1, a Kruppel/Krox gene from Strongylocentrotus purpuratus. Mech. Dev. 60, 185-195

Wilm, T. P. and Solnica-Krezel, L. (2005). Essential roles of a zebrafish prdm1 blimp1 homolog in embryo patterning and organogenesis. Development 132 393-404

Yamaji, M., Seki, Y., Kurimoto, K., Yabuta, Y., Yuasa, M., Shigeta, M. Yamanaka, K., Ohinata, Y. and Saitou, M. (2008). Critical function of Prdm14 for the establishment of the germ cell lineage in mice. Nat. Genet. 40, 1016-1022. Zeng, V., Ewen-Campen, B., Horch, H. W., Roth, S., Mito, T. and Extavour, C (2013). Developmental gene discovery in a hemimetabolous insect: de novo assembly and annotation of a transcriptome for the cricket Gryllus bimaculatus. PLOS ONE 8, e61479. 